Gdańsk 2020, Nr. 42

https://doi.org/10.26881/sgg.2020.42.11

Ewelina Damps

(Uniwersytet Gdański, Wydział Filologiczny/ Universität Gdańsk, Philologische Fakultät)

https://orcid.org/0000-0002-4924-2997

\title{
Forschungen polnischer Wissenschaftler zum deutschsprachigen Theater in Danzig
}

Im Mittelpunkt des vorliegenden Beitrags steht die polnische Forschung zum deutschsprachigen Theater in Danzig. Solche Studien wurden in Danzig sowohl von Polonisten und Theaterwissenschaftlern als auch von Germanisten, Anglisten und Skandinavisten durchgeführt. Der Artikel hat zum Ziel, den Forschungsstand und die Forschungsperspektiven aufzuzeigen.

Schlüsselwörter: Stadttheater in Danzig, Wanderbühnen, polnische Theaterwissenschaft

Research of Polish scholars on the German-language theatre in Gdansk. The main subject of this article is the Polish research on the German-language theatre in Gdańsk. Similar studies in Gdańsk have been conducted so far by Polish, German, English and Scandinavian language and literature scholars, as well as theatre scholars. The article aims at presenting the state of research and research perspectives.

Keywords: Municipal Theatre in Gdańsk, wandering theatre groups, polish theatre studies

Als eine an der Weichselmündung günstig gelegene und sich besonderer Privilegien erfreuende Hafenstadt spielte Danzig/Gdańsk seit dem ausgehenden Mittelalter eine besondere Rolle in Europa. In der Respublica Poloniae war Danzig die reichste und größte Stadt, auch seine spätere Geschichte schon als Teil des preußischen und später des deutschen Staates bestätigt die politische, wirtschaftliche, touristische, und - was daraus folgt - auch kulturelle Bedeutung der Stadt. Ein Beweis dafür ist die Entwicklung des Theaters in Danzig. Die Geschichte dieses Theaters hat ihren Ursprung im 15. und 16. Jh. und weckte schon das Interesse von deutschen Historikern, die ihre Forschungen noch vor dem Zweiten Weltkrieg veröffentlichten, z. B. von Ernst August Hagen, Johannes Bolte und Gotthilf Löschin. Die Studien von Hagen und Löschin behandelten das Thema sehr allgemein und sind dadurch lückenhaft. Bolte berücksichtigte zwar nur die ersten Jahrhunderte des Theaterwesens in Danzig, wies aber auf bedeutsame Archivalien hin, die er meist trefflich interpretierte. Auf seiner Studie basierte Gerda Gross, als sie ihre Doktorarbeit Das Danziger Theater in der ersten Hälfte des 17. Jahrbunderts (1939) schrieb. Sie erweiterte die Arbeit von Bolte um die Analyse einiger neuer Dokumente. 
Die umfangreichste historische Darstellung des Theaterlebens in Danzig wurde von Otto Rub, einem Schauspieler dieses Theaters, verfasst. Rub verpflichtete sich, die Tätigkeit des Stadttheaters zu dessen hundertjährigem Jubiläum, das am dritten August 1901 gefeiert wurde, zusammenzufassen. Seine Bearbeitung, die sich mit der Zeitperiode zwischen 1615 und 1893 beschäftigt, enthält jedoch viele Fehler, die das Gesamtbild verzerren und weitere Recherchen erheblich erschwerten. Eine wertvolle Ergänzung der Forschung ist eine umfassende Studie von Bruno Thomas Satori-Neumann, die sich ausschließlich den Theateraufführungen aus Elbing widmet. Die erste Theatergruppe, die in Danzig blieb und eine feste Bühne gründete, die Theatergesellschaft Geschwister Schuch, verfügte nämlich über eine Konzession für die Theatertätigkeit in West- und Ostpreußen: „Das Privilegium für die Geschwister Schuch“, erteilt am 3. Januar 1788 (Satori-Neumann 1936: 61). Sie spielte dementsprechend nicht nur in Danzig, sondern auch in Elbing, Marienwerder, Posen, Breslau und Königsberg.

Erwähnenswert ist auch die Studie Das Danziger Theater und sein Erbauer Carl Samuel Held von Waldemar Krause aus dem Jahre1936, der sich sowohl zur Fechtschule (dazu später mehr), als auch zum ersten Theatergebäude am Kohlenmarkt und dessen Erbauer äußerte.

$\mathrm{Zu}$ den neueren und gegenwärtigen polnischen Erforschern der Danziger Theatergeschichte gehören folgende Wissenschaftler (alphabetisch geordnet): Jan Ciechowicz, Zenon Ciesielski, Ewelina Damps, Piotr Kąkol, Edmund Kotarski, Jerzy Limon, Zbigniew Raszewski, Anna Świderska und Tadeusz Witczak. Sie beschrieben seit den 1950er Jahren vor allem die Beziehungen zwischen dem deutschen Theater in Danzig und dem polnischen Theater und konzentrierten sich dabei entweder auf einzelne Theatergruppen oder auf wichtige Ereignisse aus dem Danziger Theaterleben. Auf diese Weise entstand übergreifendes, wenn auch noch nicht vollständiges Bild von der Geschichte der Danziger Bühne.

\section{Forschung zum Wandertheater im 16. und 17. Jh.}

Hauptsächlich drei polnische Wissenschaftler, Tadeusz Witczak, Edmund Kotarski und Jerzy Limon, behandelten das Thema der Geschichte des Danziger Theaters im 16. und 17. Jh.

Tadeusz Witczak, Literaturwissenschaftler (Skandinavist) an der Universität in Posen (UAM), veröffentlichte im Jahr 1959 die Studie Teatr i dramat staropolski w Gdańsku. Im 15. und 16. Jh. wurden nur Turniere, Tänze und Gelegenheitsveranstaltungen von verschiedenen Bruderschaften veranstaltet. Im 16. Jh. fanden die Aufführungen sowohl auf den Straßen als auch in dazu adaptierten Gebäuden statt, u. a. in der Fechtschule. Witczak ging davon aus, dass Wandertheatergruppen aus England und aus Deutschland im Danzig des 16. Jh.s auftraten. Als erste kam im Jahre 1587 die englische Gruppe (Witczak 1959: 9). Der Autor beschrieb das Schaffen polnischer Autoren (Witczak 1959: 30-64) und den Aufenthalt von Luisa Maria Gonzaga, der Ehefrau von Władysław IV., im Jahr 1646 in Danzig, wo in der Fechtschule eine Oper für sie aufgeführt wurde (Witczak 1959: 68-80).

Edmund Kotarski, Polonist an der Universität Gdańsk, betonte die Bedeutung der Stadtbewohner und des Stadtrates in Gdańsk für die Entwicklung der Kultur und des Theaters (Kotarski 1988: 8). Ferner wies er irrtümlich darauf hin, dass die erste Fechtschule, in der Aufführungen gespielt wurden, bereits Ende des 16. Jh.s eröffnet wurde, um 1600 entstand 
die nächste Fechtschule, die 1635 von Logen oder Galerien umgeben und mit einem Dach bedeckt wurde. Theateraufführungen fanden auch im Gymnasium in Gdańsk statt. Anlässe zu Auftritten boten sowohl der Karneval (Februar/März) als auch der Dominikaner Markt, der in der Regel jährlich vom fünften August bis Ende August stattfand. Wie Kotarski feststellte, traten wandernde Theatergruppen aus England, Holland und Deutschland in Gdańsk auf, die vom Stadtrat für einige Tage (meist 8-14 Tage, einige Gruppen blieben länger, d. h. 6 bis 8 Wochen) ein Privileg für die Auftritte bekamen (Kotarski 1988: 8-9). Er listete die Leiter bestimmter Ensembles und die Daten ihrer Auftritte sowie teilweise ihr Repertoire auf. Unter den deutschen Künstlern erwähnte er die Truppe von Karl Andreas Pauli aus Hamburg, die 1669 für den Dominikaner Markt nach Gdańsk kam und einige Monate blieb. Die Gruppe war bekannt für ihre Auftritte in Deutschland, in der Schweiz und in Schw den (Kotarski 1988: 9). Die Besuche der ausländischen Wandertheater ermöglichten den Zuschauern, Repertoire und Künstler von internationalem Rang kennen zu lernen. Kotarski betonte auch die Bedeutung des literarischen Lebens in Gdańsk für die Entwicklung des lokalen polnischen Theaters. ${ }^{1}$ Die Autoren griffen nach solchen literarischen Gattungen wie Moralität, Mysterien, Dialoge oder Oper und nach biblischen sowie antiken Themen. Kotarski hob ferner die Rolle des Jesuitentheaters hervor.

Jerzy Limon, Anglist, Literatur- und Theaterwissenschaftler an der Universität Gdańsk, Vorsitzender der Stiftung Theatrum Gedanense und seit 2008 Direktor des Danziger Shakespeare-Theaters, untersuchte die Auftritte der englischen Wandertheatertruppen in Danzig zwischen 1601-1619 und 1636-1655. Die Ergebnisse seiner Forschung veröffentlichte er sowohl in einzelnen Artikeln in Pamiętnik Teatralny als auch in seiner Monographie Gentlemen of a Company. English Players in Central and Eastern Europe 1590-1660 (1985), dem Artikel „Zapomniana rocznica 400-lecia pierwszej wizyty aktorów angielskich w Gdańsku“ von 1988 sowie in der ein Jahr später erschienenen Monographie Gdański teatr elżbietański. Auf der Basis der erhaltenen Dokumentation konnte er sowohl die Rollenbesetzung wie auch das Repertoire erforschen. Auch laut Limon war der jedes Jahr im August stattfindende Dominikaner Markt ein wichtiger Anlass für Theaterauftritte. Limon wies jedoch darauf hin, dass die Theatergruppen aus dieser Zeit eine große Rotation von Schauspielern auszeichnete und dass sich in dem Fall keine festen Schauspielergesellschaften identifizieren lassen. Thema seiner Studie war auch das im 17. Jh. erbaute Gebäude in Danzig, die sogenannte Fechtschule, die vor allem den Schauspielern zur Verfügung stand. Als Muster für die Fechtschule diente das Fortune Theatre in London, d. h. das Konzept der offenen, elisabethanischen Bühne wurde in Danzig direkt übernommen. Limon verglich die beiden Theatergebäude miteinander im Hinblick auf ihre Architektur. Diese Fechtschule war laut Limon das erste öffentliche Theater in Polen und eines der ersten in Mitteleuropa (Limon 1977: 148).

1 Folgende literarische Werke wurden im Artikel von Kotarski genannt: „Tragedyja ucieszna albo komedyjka dworska o pijanicy, co królem był”; „Tragedyja o bogaczu i Łazarzu z Pisma Świętego wyjęta i nowo wierszem opisana polskim. Jaśnie Wielmożnemu Senatowi Gdańskiemu dedykowana i przypisana in honorem roku 1643 miesiąca stycznia dnia 22”; „Historyja o starem i młodem Tobijaszu”. 
Witczak, Kotarski und Limon bahnten so den Weg für weitere Studien. Sie erwähnten ebenfalls deutschsprachige, niederländische und schwedische Wandertheatertruppen. Deren Geschichte wurde bisher nicht erforscht und harrt noch der Aufarbeitung.

Erwähnenswert ist auch Jerzy Marian Michalak, Pianist, Dirigent, Pädagoge, der unter dem Titel „Nowe spojrzenie na gdański ,teatr elżbietański i i jego budowniczego“ im Jahr 2000 eine Kontroverse mit Jerzy Limon und zugleich auch mit Edmund Kotarski veröffentlichte. Er bezog sich auf unpräzise Datenangaben von Limon und bewies, dass die ersten Wandertheater in der alten Fechtschule auftraten, deren Entstehungsdatum unbekannt ist: Es lässt sich nur feststellen, dass der Eigentümer seine Einnahmen in der Mitte der 90er Jahre des 16. Jh.s in der Stadtkasse verbuchte. Die neue Fechtschule wurde laut Michalak erst 1635 von Jakub van dem Block erbaut und es ist dieses Gebäude, das ein Stich von Peter Willer $(1650)^{2}$ darstellt. Seine Forschung bestätigt zudem den Aufenthalt von Maria Antonina Gonzaga in Danzig im Jahre 1646, an dem andere Wissenschaftler zweifelten.

Jerzy Marian Michalak ist auch der Autor einer Publikation mit dem Titel Od Förstera do Früblinga. Przyczynki do dziejów życia muzycznego i teatralnego dawnego Gdańska aus dem Jahr 2009. Auf Deutsch erschienen seine Aufsätze zur Musik- und Theatergeschichte Danzigs vom 17. bis zum 20. Jahrbundert einige Jahre später. Michalak konzentriert sich überwiegend auf Musiker, auf ihr Leben und Wirken, zugleich berührt er das Thema der Theaterkonzerte und der Opern, insbesondere der italienischen, die auf der Danziger Bühne präsentiert wurden. Er erarbeitete sogar die Spielpläne der Konzerte, wodurch eine breite Repertoireübersicht entstand.

\section{Das Theater im 18. Jh. im Spiegel der Theaterzettel}

Eine neue Forschungsperspektive eröffnete Piotr Kąkol, der in seiner Dissertation die Tätigkeit dieses Berufstheaters (Wandertruppen) in Danzig anhand der Theaterzettel untersuchte. Seine wissenschaftlich gründlich fundierte Abhandlung besteht aus zwei Teilen. Im ersten Teil charakterisierte er die Ereignisse aus der Geschichte des Danziger Theaters von 1730 bis 1800. Der erste Theaterzettel aus dem 18. Jh. stammt nämlich aus dem Jahr 1730 und enthält die Ankündigung der Aufführung „auf einem gantz neuen Theatro“ (Kąkol 2009: 17), d. h. in der modernisierten Fechtschule.

Die ausgewerteten Theaterzettel boten die Möglichkeit, einen Abriss der Geschichte des Theaterlebens in Danzig darzustellen. Einige deutsche Wandertheatertruppen besuchten Danzig im 18. Jh. regelmäßig und spielten in der Fechtschule. Gemeint sind die Gruppen von Martin Müller und Johann Carl Kreutzer, Konrad Ernst Ackermann, Carl Theophil Döbbelin, Franz Schuch dem Älteren und dem Jüngeren sowie Caroline Schuch. Vier Theaterzettel veranschaulichen die Wirkung des Danziger Goldschmidts Johann Carl Dietrich als Theaterimpresario. Kąkol beschäftigte sich außerdem mit der Tätigkeit des Puppenspieltheaters, das im 18 Jh. in Danzig wirkte. Den zweiten Teil seiner Dissertation widmete er dem

2 Der Stich wurde zum ersten Mal im Werk Der Stadt Dantzigk historische Beschreibung von Reinhold Curicke aus dem Jahr 1687 reproduziert. 
Theaterzettel selbst, d. h. dessen Rolle als Informationsträger im Stadtraum. Er berücksichtigte dabei solche Aspekte wie Stadtbehörde, Zensur, Druck und Austragen der Anzeigen. Kąkol berührte auch methodische Probleme, wie etwa die Fehldatierung der Theaterzettel, insbesondere aus der zweiten Hälfte des 18. Jh.s. Darüber hinaus besprach er die inhaltliche Struktur und ihre Entwicklung im Laufe der Zeit. Die Dissertation wurde durch zwei Anhänge ergänzt: Der erste enthielt das Repertoire der in Danzig spielenden Wandertheater, der zweite (auf einer CD) umfasste eine Aufstellung der Danziger Theaterzettel aus dieser Zeitperiode (insgesamt 665 Exemplare) und bildete zugleich den wesentlichen Teil von Kąkols Forschung. Diese Studie zeichnete sich durch ihren innovativen Charakter und eine detaillierte, gründliche Analyse der überlieferten Artefakte aus. Seine Problemstellung ermöglichte es Kąkol, das vorhandene Quellenmaterial mit älteren deutschsprachigen Forschungen kritisch zu vergleichen.

\section{Das Theater im Danzig unter Preußens Oberherrschaft}

,Der Vater der polnischen Theaterwissenschaft' Zbigniew Raszewski veröffentlichte 1955 seine Monographie $Z$ tradycji teatralnych Pomorza, Wielkopolski i Śląska (1955). Er verdeutlichte den polnischen Lesern den damals aktuellsten Stand der Wissenschaft über die Wirkung des polnischen Theaters auf diesem Gebiet und bezog sich dabei auf die Gastauftritte der polnischen Theatergruppen von Józef Nowicki (1793), Tomasz Truskolaski (1797), Le Doux (1804) und Bogusławski (1811). Übrigens waren die Theaterzettel der Auftritte von Nowicki und Truskolaski schon im Jahr 1955 bekannt. Als Nächstes beschrieb Raszewski das Gastspiel des Polnischen Theaters aus Posen in Zoppot in den Jahren 1892-1894 und in Danzig im Jahr 1893. Ferner beschäftigt er sich mit dem polnischen Amateurtheater in Danzig aus der 2. Hälfte des 19. Jh.s - gemeint ist hier der Verein „Ogniwo“ [Kettenglied] (Raszewski 1955: 85-91).

Raszewski war ein Wegbereiter für die spätere, noch gründlichere Erforschung des polnischen Theaters in den damals deutschen Gebieten. Seine Vorliebe für Theaterforschung, die sorgfältige Analyse kleinster Details und ein zugänglicher Schreibstil prägten die nächste Generation polnischer Theaterwissenschaftler.

1967 erschien noch der bedeutende Beitrag „Teatr Bogusławskiego w Gdańsku. Odszukane afisze baletu (1804) i zespołu dramatycznego (1811)" von Zenon Ciesielski und Anna Świderska. Er beschäftigt sich mit den wiedergefundenen Theaterzetteln von den Ballettaufführungen der Truppe des Herrn Le Doux und den Aufführungen der Truppe von Wojciech Bogusławski in Danzig. Der Ballettmeister Le Doux aus Warschau gab vermutlich 10 Auftritte in Danzig, die Theaterzettel bestätigen sieben von ihnen. Wahrscheinlich dauerte sein Aufenthalt in der Stadt mindestens zwei Monate - vom 18. Juni bis zum18. August 1804. Von Bogusławskis Auftritten sind vier Theaterzettel erhalten geblieben. Bemerkenswert ist, dass sie in drei Sprachen gedruckt wurden: Polnisch, Französisch und Deutsch.

Zur weiteren Entwicklung der wissenschaftlichen Erforschung der lokalen Bühne trug vor allem Jan Ciechowicz, ein Polonist und Theaterwissenschaftler der Universität Gdańsk bei. Ciechowicz konnte selbst zwar kein Deutsch, er ermunterte aber andere Wissenschaftler zu diesen 
Forschungen und übernahm die Redaktion bedeutender wissenschaftlicher Veröffentlichungen zu dem Thema. 1988 erschien der Sammelband Gdańsk teatralny. Historia i wspótczesność unter seiner Redaktion in Zusammenarbeit mit Halina Kasjaniuk. Über die Geschichte des Danziger Theaters wurden in dem Band drei Artikel publiziert: darunter die zwei bereits besprochenen von Edmund Kotarski und Jerzy Limon. Der dritte ist „Życie teatralne w Gdańsku 1815-1920“von Bogdan Czyżak, einem Literaturhistoriker aus Danzig. Er stellte ein fragmentarisches Bild der Danziger Bühne dar und ging dabei von den Auftritten der polnischen Künstler in Danzig aus, z. B. von Nowicki (1792), Truskolaski (1797), Bogusławski (1811) und Eugeniusz Majdrowicz (1892). Darüber hinaus beschrieb er die Geschichte des polnischen Amateurtheaters der Vereine "Ogniwo" und "Jednośćc [Einheit] (Czyżak 1988: 32-36). Ohne große Erklärung begann Czyżak die Darstellung der Geschichte der deutschen Bühne mit dem Jahr 1815 und mit der Direktion von Daniel Huray, obwohl die deutsche Bühne am Kohlenmarkt bereits 1801 eröffnet wurde. Er äußerte sich in seinem Artikel kurz zu jeder Direktion dieser Bühne. Sein besonderes Interesse weckte sowohl die langjährige Theaterleitung durch Friedrich Genée (1841-1855) als auch die nicht verwirklichten Baupläne eines neuen Theatergebäudes in Danzig, das zum 100-jährigen Bestehen des Theaters erbaut werden sollte. Er bezog sich hier auf die Broschüre von Carl Fuchs Die Danziger Theaterfrage an der Wende des Jahrbunderts aus dem Jahr 1899. Anstelle eines neuen Theaters wurde aber die Technische Hochschule in Danzig erbaut. Czyżak basierte auf den früheren Bearbeitungen von Witczak, Raszewski und Rub, ohne weitere eigene Studien durchzuführen. Er betonte den provinziellen Charakter dieser Bühne sowie das immer niedrigere Niveau des Repertoires:

Das Stadttheater driftete, vor allem seit der Einigung Deutschlands in eine feindliche, vom Chauvinismus geprägte Isolation ab. Solange der universelle Trend der alten Kultur der Stadt Gdańsk lebendig war, war die Bühne offen für neue Muster. Die Provinzialisierung des Theaters spiegelte sich in der Tatsache wider, dass es seine anachronistische Mehrzweckformel bis zum Ende seiner Existenz beibehielt, sowie in seiner fortschreitenden künstlerischen Bedeutungslosigkeit. Die lange Abwesenheit des Polnischen als Ergebnis der falschen Ansicht über den Fortschritt der Germanisierung in Gdańsk war ein irreparabler Schaden. Im Widerspruch dazu stand die sehr lebendige polnische Theaterbewegung der 70er Jahre und der folgenden Jahrzehnte. Zu diesem Zeitpunkt war Gdańsk jedoch bereits zu einer Arena für einen rücksichtslosen Kampf gegen die polnische Wesensart geworden, die einst die treibende Kraft ihrer kulturellen Entwicklung war (Czyżak 1988: 43) [Übersetzung: Ewelina Damps].

Um zu Jan Ciechowicz zurückzukehren: Als Experte für das Theater des 19. Jh.s untersuchte er die Beziehungen zwischen dem Theater in Danzig und dem polnischen Theater in dieser Zeit. In seiner Monographie Myślenie teatrem hebt sich das Kapitel „Das Theater in Danzig und seine Umgebung" heraus, wo er u. a. die Thesen eines früheren Artikels von Zbigniew Raszewski über die Auftritte der Theatertruppe Wojciech Bogusławskis im Jahre 1811 in Gdańsk weiterentwickelte. Ciechowicz griff nach einigen neuen Details und Interpretationsversuchen, darunter nach dem Rocznik Teatru Narodowego Księstwa Warszawskiego und der Gazeta Warszawska. Er beschäftigte sich damit erneut mit dem von Bogusławski vorgeschlagenen Repertoire und seinen Auftritten in Danzig. Der Schreibstil von Ciechowicz zeichnet sich durch erzählerische Leichtigkeit und eine zuverlässige Analyse von Fakten aus. Ciechowicz' breites Spektrum an Theaterkenntnissen erlaubte es ihm auch, Zusammenhänge zwischen dem polnischen und deutschen Theater aufzuzeigen. 
Aus Anlass des zweihundertjährigen Jubiläums des Danziger Stadttheaters erschien im Jahr 2004 unter der Redaktion von Jan Ciechowicz der Sammelband 200 lat teatru na Targu Weglowym $w$ Gdańsku, dessen Kapitel die Überschriften tragen: Probleme der Architektur, Wo soll man nach Quellen suchen?, Beginn und Entwicklung, Schwieriges Erbe, Gedächtnis und Dauern, Um Hanemann, und Bilder und Menschen. Das letzte Kapitel ist eine Sammlung von Illustrationen, die von Małgorzata und Mieczysław Abramowicz für eine Ausstellung ausgewählt wurden. Zur Zusammenarbeit lud Ciechowicz Experten aus dem Bereich der Theaterarchitektur (Hans-Peter Bayerdörfer, Robert Hirsch, Andrzej Prusiewicz, Elżbieta Ratajczak-Piątkowska, Jakub Szczepański, Lech Kadłubowski, Stanisław Fiszer), Mitarbeiter der Polnischen Akademie der Wissenschaften (PAN) in Gdańsk (Maria Babnis), des Staatsarchivs in Gdańsk (Katarzyna Komsta) sowie der Freien Universität Berlin (Dagmar Walach), Theater-, Literatur- und Musikwissenschaftler (Piotr Kąkol, Mieczysław Abramowicz, Małogorzata Leyko, Jerzy Marian Michalak, Peter Oliver Loew, Katarzyna PrykowskaMichalak, Katarzyna Weiss, Stephan Wolting, Bogusław Drewniak, Hanna Dyktyńska, Andrzej Wirth, Joanna Chojka) und den damaligen Leiter des Theaters Wybrzeże Maciej Nowak ein.

Die Publikation, die eine Konferenz zusammenfasste, warf ein neues Licht auf die Forschung und eröffnete ihr weitere Perspektiven. Architekturexperten verglichen das 1801 am Kohlenmarkt eröffnete Theaterhaus mit anderen Projekten aus dieser Zeit sowie mit Folgeprojekten aus den Jahren 1935 und 1967 und versuchten, das von Carl Samuel Held erbaute Gebäude als Ort öffentlicher Veranstaltungen zu interpretieren. Vertreter der Institutionen, die Theaterarchivalien aufbewahren, wiesen auf vorhandene Quellen hin, wie z. B. auf die Sammlung von Theaterzetteln oder die Theatersammlung von Walter Unruh an der Freien Universität Berlin, die noch neue Forschungsmöglichkeiten bieten. Mieczysław Abramowicz und Małgorzata Leyko versuchten, die Ereignisse vom dritten August 1801, dem Tag der Eröffnung des Theaters, im Detail zu rekonstruieren. Der Artikel von Jerzy Marian Michalak enthielt eine detaillierte Untersuchung des Opern- und Ballettrepertoires zur Zeit der Direktion Friedrich Genées (1841-1855). Darüber hinaus erschienen Referate über literarische Texte, etwa über Max Halbe, und den Einfluss der politischen und nationalen Situation der Freien Stadt Danzig auf das Theater (1920-1939).

Dank dem Engagement von Ciechowicz enthält auch die Encyklopedia Gdańska Einträge über das Danziger Theaterleben. Zu ihren Autoren gehören: Piotr Kąkol, Ewelina Damps, Eliza Szymańska und Jan Ciechowicz (nach 1945).

In meiner Dissertation Historia Teatru Miejskiego w Gdańsku (1801-1841) von 2015 habe ich die ersten 40 Jahre des ständigen Theaters in Danzig untersucht. Die Monographie besteht aus drei Kapiteln, die chronologisch angeordnet sind. Zuerst habe ich die ersten Wirkungsjahre dieses Theaters besprochen, und zwar mit besonderer Berücksichtigung der Architektur, der Entstehung des neuen Theatergebäudes und aller wichtigen Ereignisse sowie der hervorragenden Schauspieler unter den Direktionen von Carl Steinberg und Jean Bachmann. Das nächste Kapitel ist der Versuch einer Charakteristik der , besonderen Ehe' zwischen Kunst und Politik, d. h. des Einflusses der Franzosen auf das Repertoire und die Wirkung des Theaters in der ersten Freien Stadt Danzig (1807-1814). Das dritte Kapitel enthält Informationen über die Zeit, in der die Direktoren ziemlich häufig wechselten und die ökonomische 
Situation des Theaters keine Verbesserungen ankündigte. ${ }^{3}$ In diesem Teil der Dissertation habe ich folgende Themen besprochen: den technischen Zustand des Theaters, die Entwicklung der Presse und der Theaterkritik, die Dramatikerinnen im Danziger Theaterrepertoire.

\section{Forschungen über das Theater in der Freien Stadt Danzig}

Zenon Ciesielski publizierte 1969 seine Arbeit Teatr polski w Wolnym Mieście Gdańsku 1920-1939, in der er alle Orte, die von polnischen Amateurtheatern als Theaterbühnen benutzt wurden, sowie die einzelnen Gruppen und ihre Tätigkeit charakterisierte. Ferner besprach er die Gastauftritte polnischer Künstler in Danzig, u. a. des Nationaltheaters aus Thorn (das mehrmals spielte), des Polnischen Theaters aus Posen, der Wanderoper aus Warschau, des Theaters Reduta, die Zusammenarbeit mit dem Theater in Bromberg und in Gdingen. Der Anhang enthält eine Liste von insgesamt 349 Theateraufführungen und 149 Gastspielen. Ciesielski betonte den starken Widerstand der städtischen Behörden gegen diese Gastspiele und zugleich das Interesse seitens der deutschen Einwohner.

Im Jahr 2003 veröffentlichte der in Polen ansässige deutsche Literaturwissenschaftler Stephan Wolting seine Habilitation Bretter, die Kulturkulissen markierten. Das Danziger Theater am Koblenmarkt, die Zoppoter Waldoper und andere Theaterinstitutionen im Danziger Kulturkosmos zur Zeit der Freien Stadt und in den Jahren des Zweiten Weltkriegs. Er behandelt darin zuerst kurz das kulturelle Leben in Danzig, danach die Frage des Eigentums des Theatergebäudes und das Wilhelmstheater (eine Unterhaltungsbühne), konzentriert sich aber auf das Danziger Stadttheater (ab 1935 Staatstheater) und dessen Repertoire. Darauf folgt ein rund 50 Seiten langer Teil zum Personal am Danziger Stadttheater. Aufschlussreich sind Woltings Ausführungen über die Zoppoter Waldoper und seine Interpretation der Dokumente aus dem Politischen Archiv des Auswärtigen Amtes in Berlin, denn diese geben Aufschluss über die Finanzierung der Waldoper durch das Deutsche Reich seit den 1920er Jahren. Die Arbeit enthält noch einen Anhang mit dem Spielplan des Stadttheaters zwischen 1918 und 1944. Woltings Habilitation stieß leider auf starke Kritik. So wies der deutsche Historiker Peter Oliver Loew u. a. auf Fehler hin, die ihren Wert herabmindern (Loew 2006: 309-314).

\section{Forschungsdesiderate}

Trotz vieler wissenschaftlicher Arbeiten über das Danziger Theater lassen sich immer noch Themenbereiche aus der Geschichte dieser Bühne entdecken, die es wert sind, ausführlichen, wissenschaftlichen Studien unterzogen zu werden. Dazu gehören: Auftritte der deutschsprachigen, niederländischen und schwedischen Wandertheatertruppen im Danzig des 16. und 17. Jh.s und die Ereignisse aus der Geschichte des Theaters von 1841 bis 1918. Sinnvoll wäre

3 Zu den Direktoren gehörten Bernhard Kohler (1819-1820), Adolph Schröder (1820-1831), Johann Huray (1831-1834), Eduard Dohring (1834-1836), August von Zieten (1836-1837), Anton Hübsch (1837-1838) und Gustav Heinrich Leopold Laddey (1838-1841). 
es, eine weitere und detaillierte Erforschung der Geschichte dieses Theaters nach dem Jahr 1918 durchzuführen. Obgleich sich das Theater in Danzig unter den Wissenschaftlern einer großen Beliebtheit erfreute, bleibt also noch einiges zu tun.

\section{Literatur}

Bolte, Johannes (1895): Das Danziger Theater im 16. und 17. Jahrhundert. In: Theatergeschichtliche Forschung. 12. Hamburg, Leipzig: Leopold Voss.

Ciechowicz, Jan (2000): Bogusławski w Gdańsku [Bogusławski in Danzig]. In: ders.: Myślenie teatrem [Mit dem Theater denken]. Gdańsk: Wydawnictwo Uniwersytetu Gdańskiego, 257-270.

Ciechowicz, Jan (Hg.) (2004): 200 lat teatru na Targu Weglowym w Gdańsku [200 Jahre Theater am Kohlenmarkt in Danzig]. Gdańsk: Nadbaltyckie Centrum Kultury, Uniwersytet Gdański.

Ciechowicz, Jan / Kasjaniuk, Halina (Hg.) (1988): Gdańsk teatralny. Historia i wspótczesność [Theatralisches Danzig. Geschichte und Gegenwart]. Gdańsk: Państwowy Teatr Wybrzeże.

Ciesielski, Zenon (1969a): Niemieckie teatry Wolnego Miasta Gdańska i ich stosunek do społeczeństwa polskiego. In: Rocznik Gdański. 28, 53-76.

Ciesielski, Zenon (1969b): Teatrpolski w Wolnym Mieście Gdan'sku 1920-1939 [Das polnische Theater in der Freien Stadt Danzig 1920-1939]. Gdańsk: Wydawnictwo Morskie Gdańsk.

Ciesielski, Zenon / Świderska, Anna (1967): Teatr Bogusławskiego w Gdańsku. Odszukane afisze baletu (1804) i zespołu dramatycznego (1811) [Das Theater von Bogusławski in Danzig. Die wiedergefundenen Theaterzettel des Balletts (1804) und des dramatischen Ensembles (1811)]. In: Pamiętnik Teatralny. 3/4, 458-462.

Curicke, Reinhold (1687): Der Stadt Dantzigk historische Beschreibung. Danzig, Amsterdam: Verlegt durch Johann u. Gillis Janssons von Waesberge.

Czyżak, Bogdan (1988): Życie teatralne w Gdańsku 1815-1920. [Das Theaterleben in Danzig 1815-1920]. In: Ciechowicz, Jan / Kasjaniuk, Halina (Hg.): Gdańsk teatralny. Historia i wspótczesność. Gdańsk: Państwowy Teatr Wybrzeże, 30-43.

Damps, Ewelina (2015): Historia Teatru Miejskiego w Gdańsku (1801-1841). [Die Geschichte des Stadttheaters in Danzig (1801-1841)]. Gdańsk: Wydawnictwo Uniwersytetu Gdańskiego.

Gross, Gerda (1939): Das Danziger Theater in der ersten Hälfte des 17. Jahrhunderts. In: Kindermann, Heinz (Hg.): Danziger Barockdichtung. Leipzig: Reclam, 266-333.

Hagen, Ernst August (1854): Geschichte des Theaters in Preußen vornämlich der Bühne in Königsberg und Danzig von ihren ersten Anfängen bis zu den Gastspielen J. Fischer's und L. Devrient's. Königsberg: E. I. Dalkowski.

Kąkol, Piotr (2009): O teatrze zawodowym w osiemnastowiecznym Gdanisku. Wokót gdańskiego afisza. [Über das Berufstheater im Danzig des 18. Jahrhunderts. Rund um den Danziger Theaterzettel]. Gdańsk: Wydawnictwo Uniwersytetu Gdańskiego.

Kotarski, Edmund (1988): Teatr i dramat w siedemnastowiecznym Gdańsku [Theater und Drama im Gdańsk des 17. Jahrhunderts]. In: Ciechowicz, Jan / Kasjaniuk, Halina (Hg.): Gdańsk teatralny. Historia i wspótczesność. Gdańsk: Państwowy Teatr Wybrzeże, 8-21.

Krause, Waldemar (1936): Das Danziger Theater und sein Erbauer Carl Samuel Held. Danzig: Kafemann. 
Limon, Jerzy (1985): Gentlemen of a Company. English Players in Central and Eastern Europe 1590-1660. Cambridge: Cambridge University Press.

Limon, Jerzy (1989): Gdański teatr elżbietański. [Das Danziger elisabethanische Theater]. Wrocław, Warszawa, Kraków, Gdańsk, Łódź: Zakład Narodowy im. Ossolińskich.

Limon, Jerzy (1977): Przypuszczalne związki teatru gdańskiego „Szkoły Fechtunku” z teatrem „Fortune” w Londynie. [Die vermutlichen Zusammenhänge der Danziger Fechtschule mit dem FortuneTheater in London]. In: Pamiętnik Teatralny. 1, 29-39.

Limon, Jerzy (1988): Zapomniana rocznica 400-lecia pierwszej wizyty aktorów angielskich w Gdańsku. [Das vergessene 400-jährige Jubiläum des ersten Besuchs englischer Schauspieler in Gdańsk]. In: Ciechowicz, Jan / Kasjaniuk, Halina (Hg.): Gdańsk teatralny. Historia i wspótczesność. Gdańsk: Państwowy Teatr Wybrzeże, 22-29.

Löschin, Gotthilf (1837): Danziger Theaterwesen um die Mitte des vorigen Jahrhunderts. In: ders. Beiträge zur Geschichte Danzigs und seiner Umgebung. 1. Danzig: Wedelschen Buchdruckerei.

Loew, Peter Oliver (2006): Stephan Wolting: Bretter, die Kulturkulissen markierten. Das Danziger Theater am Kohlenmarkt, die Zoppoter Waldoper und andere Theaterinstitutionen im Danziger Kulturkosmos zur Zeit der Freien Stadt und in den Jahren des Zweiten Weltkriegs. Wrocław: Wydawnictwo Uniwersytetu Wrocławskiego 2003, 686 S. Abb. In: Studia Germanica Gedanensia. 14, 309-314.

Michalak, Jerzy Marian (2012): Aufsätze zur Musik-und Theatergeschichte Danzigs vom 17. bis zum 20. Jahrbundert. Berlin: Frank \& Timme.

Michalak, Jerzy Marian (2009): Od Förstera do Frühlinga. Przyczynki do dziejów życia muzycznego i teatralnego dawnego Gdańska. [Von Förster bis Frühling. Beiträge zur Geschichte des musikalischen und theatralischen Lebens im alten Danzig]. Gdańsk: Wydawnictwo Akademii Muzycznej im. S. Moniuszki.

Michalak, Jerzy Marian (2000): Nowe spojrzenie na gdański „teatr elżbietański” i jego budowniczego. [Eine neue Sicht auf das Danziger „elisabethanische“ Theater und seinen Erbauer]. In: Gdański Rocznik Kulturalny. 19, 107-122.

Raszewski, Zbigniew (1955): Z tradycji teatralnych Pomorza, Wielkopolski i Śląka. [Aus den Theatertraditionen in Pommern, Großpolen und Schlesien]. Wrocław: Zakład im. Ossolińskich.

Rub, Otto (1894): Die dramatische Kunst in Danzigvon 1615 bis 1893. Danzig: Verlag von Theodor Bertling.

Satori-Neumann, Bruno Thomas (1936): Dreihundert Jabre berufsständisches Theater in Elbing. Die Geschichte einer ostdeutschen Provinzialbühne. Nach den Quellen dargestellt. Bd. 1. 1605-1846. Danzig: Westpreussischer Geschichtsverein.

Śliwiński, Błażej / Mykowski, Jarosław (Hg.) (2012): Encyklopedia Gdańska. [Danziger Enzyklopädie]. Gdańsk: Fundacja Gdańska, 1030-1039.

Witczak, Tadeusz (1959): Teatr $i$ dramat staropolski $w$ Gdańsku [Das altpolnische Theater und das Drama in Gdańsk]. Gdańsk: Gdańskie Towarzystwo Naukowe.

Wolting, Stephan (2003): Bretter, die Kulturkulissen markierten: Das Danziger Theater am Koblenmarkt, die Zoppoter Waldoper und andere Theaterinstitutionen im Danziger Kulturkosmos zur Zeit der Freien Stadt und in den Jahren des Zweiten Weltkriegs. Wrocław: Wydawnictwo Uniwersytetu Wrocławskiego. 